\title{
Veränderung von Wald und Waldleistungen in der Landschaft Davos im Zuge des Klimawandels
}

\author{
Peter Bebi \\ Michaela Teich \\ Frank Hagedorn \\ Natalie Zurbriggen \\ Sibyl Hanna Brunner \\ Adrienne Grêt-Regamey
}

\author{
WSL-Institut für Schnee und Lawinenforschung $(\mathrm{CH})^{*}$ \\ WSL-Institut für Schnee und Lawinenforschung $(\mathrm{CH})$ \\ Eidgenössische Forschungsanstalt für Wald, Schnee und Landschaft $(\mathrm{CH})$ \\ Eidgenössische Forschungsanstalt für Wald, Schnee und Landschaft $(\mathrm{CH})$ \\ PLUS, Eidgenössische Technische Hochschule Zürich (CH) \\ PLUS, Eidgenössische Technische Hochschule Zürich $(\mathrm{CH})$
}

\begin{abstract}
Changes in forest cover and ecosystem services in Davos under climate change
The effects of climate change on forests of Davos were examined with field experiments near treeline, analyses of avalanche-forest interactions and with spatially explicit models for the valuation of ecosystem services. Experimental trees at the Stillberg research site showed species-specific responses to elevated $\mathrm{CO}_{2}$ and soil warming. Growth and mortality of the trees planted in the year 1975 were strongly driven by the duration of snow cover and microtopography. Together with other field studies in the region this suggests that during the next decades the treeline will rise only slowly and mainly on favorable microsites. Avalanche protection will also in future be the most important forest service in Davos, although critical weather and snow conditions for forest avalanches show a decreasing trend over the last 40 years. The density of forest structures is likely to further increase with potential positive effects on avalanche protection. Decreases of the protective effect against avalanches may however occur by an increase of natural disturbances such as fires or bark beetle outbreaks. Quantification and overlay of five selected ecosystem services (avalanche protection, recreation, $\mathrm{CO}_{2}$ sequestration and storage, habitats of capercaillie, timber production) suggest in general an increase in the value of most considered ecosystem services.
\end{abstract}

Keywords: climate change, treeline, forest cover change, avalanche protection, ecosystem services, trade-offs doi: $10.3188 /$ szf. 2012.0493

*Flüelastrasse 11, CH-7260 Davos-Dorf, E-Mail peter.bebi@wsl.ch

Z um Einfluss des Klimawandels auf kältelimitierte Waldgrenzen und Gebirgswaldökosysteme gibt es zahlreiche Felduntersuchungen und Modelle auf verschiedensten Skalen und mit zum Teil unterschiedlichen Schlussfolgerungen (Huber et al 2005). Innerhalb der «Mountland»-Region Davos (Kanton Graubünden) wurden in den letzten Jahren entsprechend dem Grundkonzept des Projektes Experimente und Beobachtungen auf kleinräumiger Skala mit Modellierungen zu Ökosystemleistungen auf grösserer Skala kombiniert, um Hinweise dazu zu erhalten, wie sich Wälder und Waldleistungen in Zukunft verändern könnten. Im Vergleich zu den beiden anderen «Mountland»-Regionen Jura (Kanton Waadt) und Visp (Kanton Wallis) sind die Wälder von Davos stärker durch Kälte und Schnee limitiert und erbringen in erster Linie wichtige Schutzleistungen gegenüber Lawinen und anderen Naturgefahren. Wir fassen einige Aspekte dieser Forschung zusammen und zeigen in diesem Beitrag auf, wie diese Wälder insbesondere an deren oberer Grenze auf eine $\mathrm{CO}_{2}$-reichere Atmosphäre und ein wärmeres Klima reagieren und wie sich deren Leistungen entsprechend verändern.

\section{Material und Methoden}

\section{Untersuchungsgebiet}

Davos ist mit rund 13000 Einwohnern und 25000 Gästebetten die höchstgelegene Stadt Europas. Mit $284 \mathrm{~km}^{2}$, welche auf einen Höhengradienten von 1080 bis $3146 \mathrm{~m}$ ü. M. verteilt sind, ist Davos auch die zweitgrösste Gemeinde der Schweiz. ${ }^{1}$ Das Klima ist gemässigt zentralalpin mit einer Jahresmitteltemperatur (auf $1560 \mathrm{~m}$ ü. M.) von rund $3.8^{\circ} \mathrm{C}$ und einer Niederschlagssumme von rund $1000 \mathrm{~mm} / \mathrm{Jahr}$. Im Talboden fällt rund 40\% des Niederschlags als Schnee (Zappa et al 2003). Ungefähr $24 \%$ der Gemeindefläche sind bewaldet, rund $65 \%$ davon sind Privatwald. Die obere Waldgrenze befindet sich auf rund $2100 \mathrm{~m}$ ü. M. und wurde zum Teil

1 www.gemeinde-davos.ch/pdf/davosinzahlen.pdf (26.9.2012) 


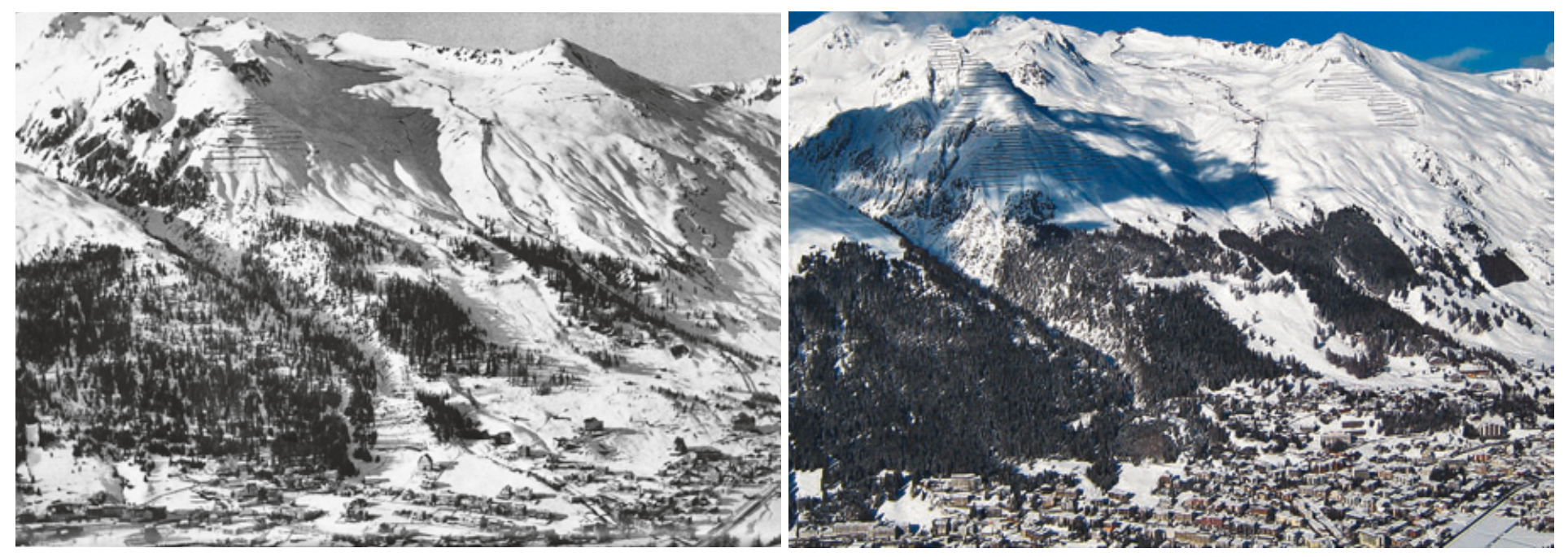

Abb 1 Schutzwald oberhalb Davos im Jahr 1945 und heute. Die Abbildungen zeigen nur einen kleinen Teil des Untersuchungsgebietes. Fotos: Foto Furter

anthropogen herabgesetzt. Wichtigste Baumarten sind Fichte (Picea abies, rund 80\% des Vorrats), Lärche (Larix decidua), Arve (Pinus cembra) und Bergföhre (Pinus mugo). Seit der Besiedlung durch die Walser im 13. Jahrhundert bis gegen Ende des 19. Jahrhunderts wurde der Wald intensiv genutzt (Beweidung, Rodung, Brenn- und Bauholz, Bergbau). Insbesondere seit dem Aufkommen des Wintertourismus nach dem 2. Weltkrieg nahm der Nutzungsdruck dann aber wieder deutlich ab, und viele Wälder wurden nicht mehr bewirtschaftet. Seit den 1980er-Jahren erlebt die Waldbewirtschaftung dank einem gut organisierten Forstbetrieb wieder einen Aufschwung, wobei das Schwergewicht auf der Pflege der Schutzwälder liegt (Abbildung 1).

\section{Langzeitbeobachtungen und Experimente an der Waldgrenze}

Die Versuchsfläche Stillberg liegt in einem typischen Lawinenanrissgebiet an der alpinen Waldgrenze und umfasst einen Höhengradienten von 2000 bis 2230 m ü. M. (Abbildung 2). Das Hauptziel des Versuchs lag zunächst darin, geeignete Verfahren für Aufforstungen in Lawinenanrissgebieten zu finden. Im Jahr 1975 wurden rund 92000 Arven, Bergföhren und Lärchen in einem regelmässigen Muster gepflanzt, und in einem Drittel der Versuchsfläche wurden temporäre Lawinenverbauungen errichtet (Schönenberger \& Frey 1988). Danach wurden regelmässig Überleben und Wachstum der Bäume erhoben (Barbeito et al 2012). In einem 2001 begonnenen Experiment wurde untersucht, wie sich der Klimawandel auf Ökosysteme an der Waldgrenze auswirkt. Je zehn Lärchen und Bergföhren wurden höheren $\mathrm{CO}_{2}$-Konzentrationen (+200 ppm) ausgesetzt, die gleiche Anzahl Bäume blieb als Kontrolle unbehandelt. Unter jeweils der Hälfte der Bäume wurde zudem der Boden während der Vegetationszeit um $3{ }^{\circ} \mathrm{C}$ erwärmt. In der Folge wurden Reaktionen des Wachstums, des Nährstoffumsatzes sowie die $\mathrm{CO}_{2}$ Bilanz des Bodens studiert (Hagedorn et al 2010, Da- wes et al 2011). Ausserdem wurde mittels eines Saatversuches auf einem Höhentransekt von 2000-2400 $m$ ü. M. untersucht, wie gut sich verschiedene Baumarten (Arve, Lärche, Fichte, Bergföhre, Waldföhre [Pinus sylvestris], Grünerle [Alnus viridis] und Vogelbeere [Sorbus aucuparia]) an und über der aktuellen Waldgrenze etablieren können und welche Faktoren dabei entscheidend sind (Moos 2012).

\section{Wechselwirkungen zwischen Wald und Lawinen}

Um Bedingungen für Lawinenanrisse im Waldbereich zu studieren, wurden seit den 1980er-Jahren Waldlawinen erfasst und die wesentlichen Standort-, Wald- und Schneeparameter erhoben (Meyer-Grass \& Schneebeli 1992, Bebi et al 2009). In den letzten Jahren wurden diese Daten ergänzt und im Hinblick auf neue Fragen ausgewertet. Dabei standen bisher vor allem drei Aspekte im Vordergrund: 1) der Einfluss von Waldstruktur, Topografie und Lawineneigenschaften auf die Reichweite einer Waldlawine (Teich et al 2012a); 2) die Entwicklung der Bestandesstruktur in ehemaligen Anrissgebieten von Waldlawinen (Ulrich 2009); 3) die Veränderung der zum Anreissen von Waldlawinen führenden Witterungsbedingungen (Teich et al 2012b).

Waldentwicklung und Ökosystemleistungen

Um die Ökosystemleistungen für die Davoser Wälder im Jahr 2000 und für unterschiedliche Szenarien bis zum Jahr 2050 zu modellieren, wurde ein iterativer Prozess zwischen GIS-basierter Abschätzung und Expertenvalidierung gewählt. Dafür wurde ein Bayes'sches Netzwerk verwendet. Dies ist ein probabilistischer Modellansatz, welcher durch Integration von neuen quantitativen Daten oder von Expertenwissen ständig verbessert wird und Unsicherheiten auf verschiedener Stufe einbeziehen kann (Grêt-Regamey et al 2012). Die zwei verwendeten Waldmodelle basieren auf Luftbildanalysen der Davoser Wälder für die Jahre 1954 und 2000 (noch 


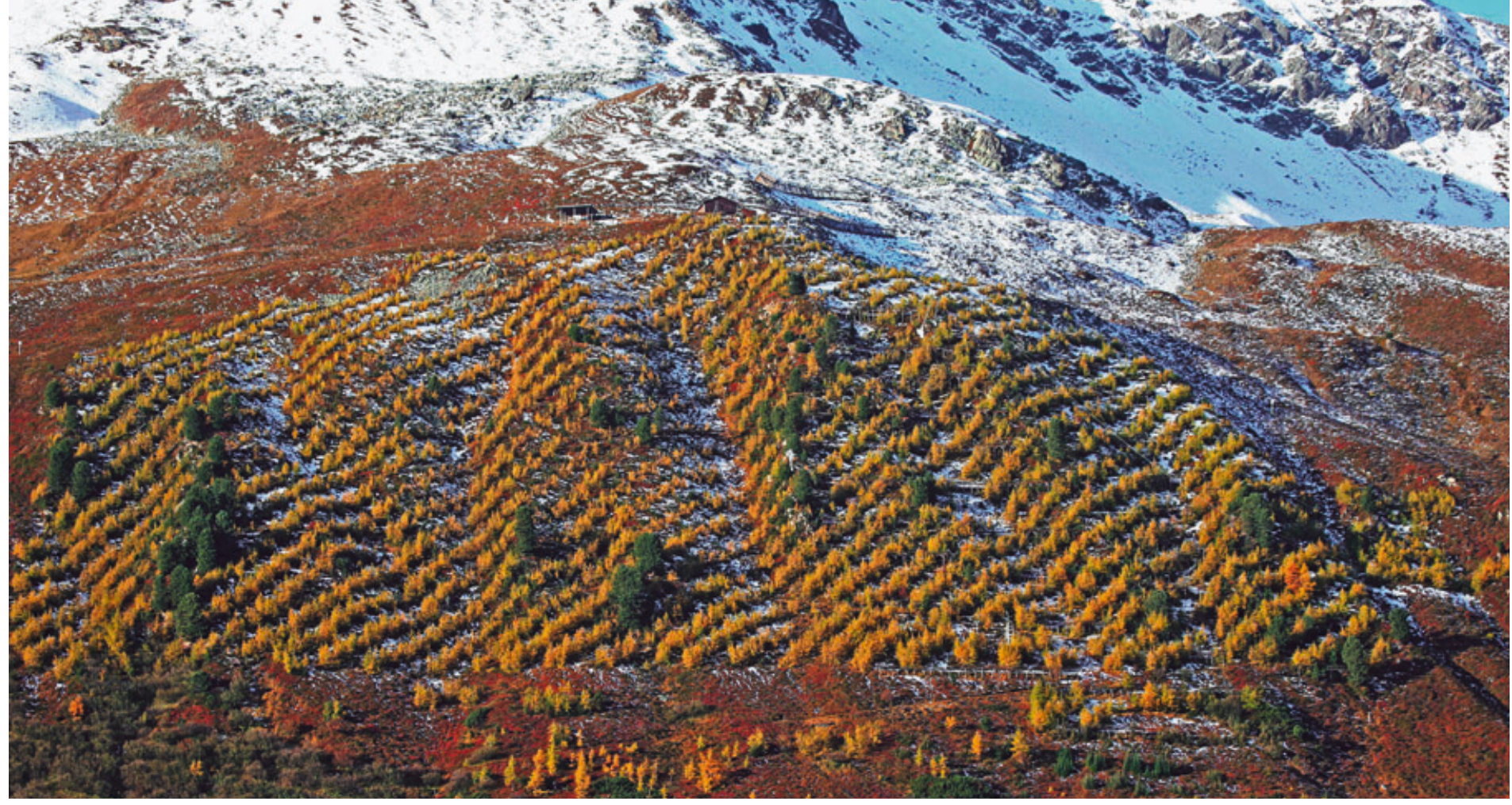

Abb 2 Versuchsaufforstung Stillberg. Von den im Jahr 1975 rund 92000 gepflanzten Bäumen lebten 30 Jahre später noch rund 30\%, wobei von den drei gepflanzten Baumarten die Lärche am besten überlebt hat. Foto: Ulrich Wasem, wSL

ohne die Fraktion Wiesen, welche erst seit 2009 zur Gemeinde Davos gehört; Lardelli 2003). Daraus wurde zunächst abgeleitet, mit welcher Wahrscheinlichkeit jeder Waldstrukturtyp in einen anderen überging, woraus dann für jede Waldfläche eine vom Waldtyp und Standort abhängige Wahrscheinlichkeitsverteilung für die zukünftige Entwicklung abgeleitet wurde. Das Trendszenario entspricht somit einer Weiterführung der bisherigen Waldentwicklung und der bisherigen Waldnutzung. Das Erwärmungsszenario beruht im Vergleich zum Trendszenario auf der Annahme, dass die Temperatur bis ins Jahr 2050 um $2.4{ }^{\circ} \mathrm{C}$ steigt (OcCC 2003, Grêt-Regamey et al 2008), was eine Erhöhung des Baumwachstums insbesondere in höheren Lagen bewirkt. Beide Szenarien wurden aufgrund von Experteninterviews, in welchen die wahrscheinliche Entwicklung der einzelnen Wälder diskutiert wurde, angepasst.

Die Abschätzung von fünf unterschiedlichen und in Davos als wichtig betrachteten Ökosystemleistungen (Lawinenschutz, Erholung, C-Speicherung, Auerhuhnhabitate und Holzproduktion) wurde mit GIS-basierten Prozessmodellen vorgenommen: Der Lawinenschutz des Waldes wurde mithilfe des 2-dimensionalen Lawinensimulationsmodells RAMMS quantifiziert (Christen et al 2011) und mit einem Risikoansatz monetarisiert (Grêt-Regamey \& Straub 2006). $\mathrm{CO}_{2}$-Senken wurden basierend auf Veränderungen des oberirdischen und unterirdischen Vorrats für verschiedene Waldstrukturen und Ableitungen von entsprechenden $\mathrm{CO}_{2}$-Speicherkapazitäten gemäss Thürig \& Schmid (2008) abgeschätzt und mithilfe von Grenzkosten der $\mathrm{CO}_{2}$-Emissionen (Nelson et al 2009) quantifiziert. Habitate für das Auer- huhn (Tetrao urogallus) wurden basierend auf dem Habitatmodell von Graf et al (2005) ermittelt und mittels eines Ersatzkostenansatzes quantifiziert (GrêtRegamey et al 2008). Die Abschätzung und Monetarisierung der Erholungsfunktion erfolgte aufgrund eines Modells, welches in erster Linie von den Faktoren «Erreichbarkeit» und «Bestandesdichte» abhängt (Bernasconi et al 2005, Beck 2008). Die Holzproduktion wurde aufgrund von potenziellen Holzerntemengen für verschiedene Waldtypen abgeschätzt und aufgrund der Differenz zwischen durchschnittlichen regionalen Marktpreisen und Erntekosten monetarisiert (Grêt-Regamey et al 2012). Die Werte der Modellvariablen und die resultierenden Ökosystemleistungskarten wurden mit je mindestens fünf Experten aus verschiedenen Disziplinen validiert.

\section{Ergebnisse}

\section{Ergebnisse der Experimente an der Wald- grenze}

Von 92000 Waldgrenzenbäumen am Stillberg (Abbildung 2) hatten 30 Jahre nach der Pflanzung rund 30\% überlebt. Bei allen drei Arten war die Mortalitätsrate zwischen dem 5. und 15. Lebensjahr am grössten, wobei der Zeitpunkt der Schneeschmelze (gutes Überleben bei Ausaperung vor Mitte Mai) während der ganzen Beobachtungsperiode der bedeutendste Faktor für die Überlebenswahrscheinlichkeit war (Abbildung 3). Im Gegensatz dazu wurde das Wachstum der Bäume am stärksten durch die Höhenlage bestimmt, es reagierte aber auch sehr sensibel auf Änderungen des Kleinstandortes und der 


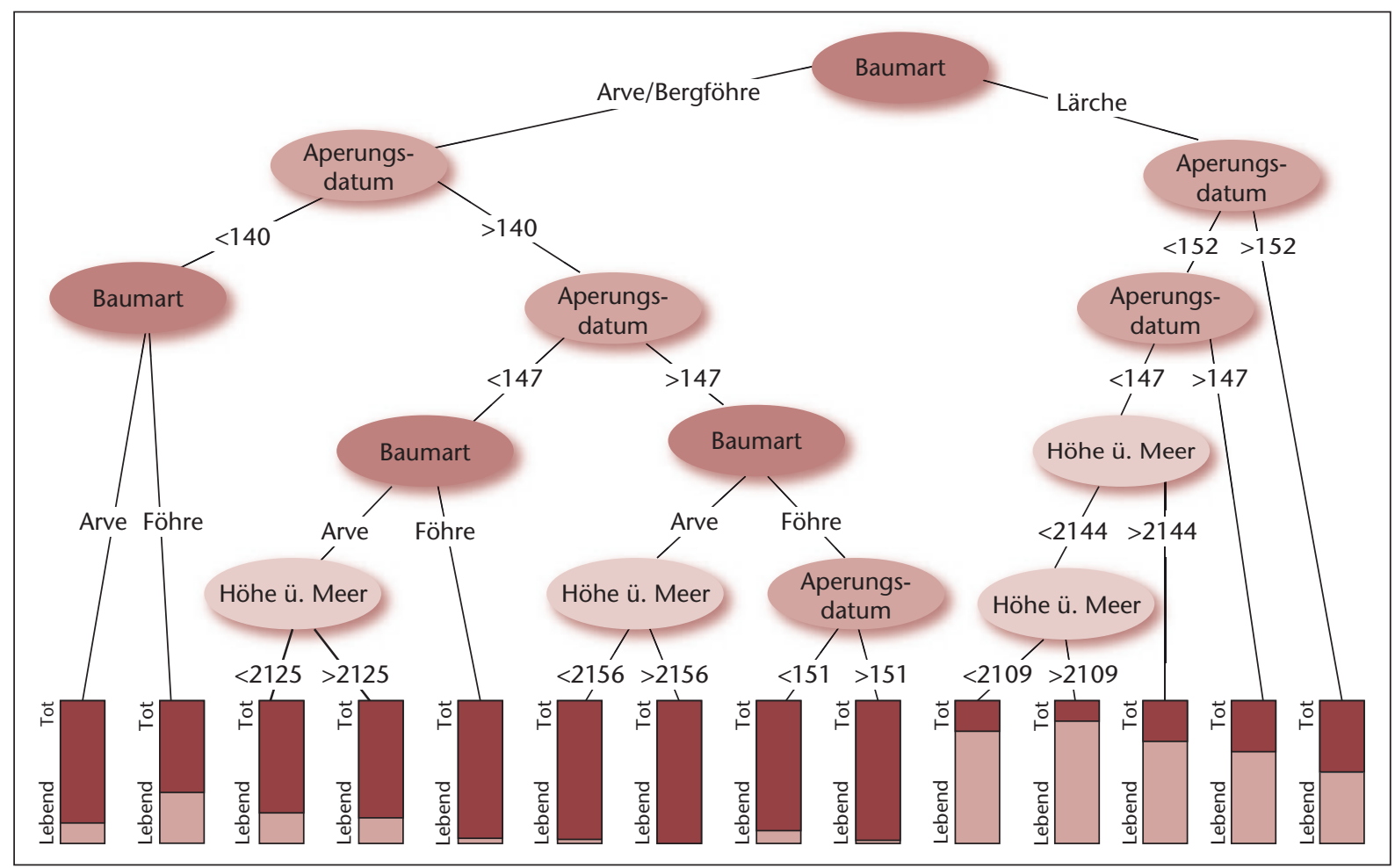

Abb 3 Überlebensfaktoren am Stillberg: Der Klassifikationsbaum teilt den Datensatz der ursprünglich gepflanzten Bäume jeweils nach dem wichtigsten Einflussfaktor auf. Die Höhe der roten Säulen zeigt für jede Faktorkombination den Anteil der abgestorbenen Bäume an. Quelle: Barbeito et al (2012)

Schneedecke. Mit zunehmender Grösse der Bäume sank der Einfluss der Einstrahlung, dafür wurden die Bäume mehr durch Wind und mechanische Beschädigungen beeinträchtigt (Barbeito et al 2012).

Mit der kleinstandörtlichen Variation (auf der Skala von wenigen Metern) kamen die gepflanzten Baumarten unterschiedlich zurecht. Die Lärche zeigte mit 69\% Überlebenden während der ersten 30 Jahre die grösste Standorttoleranz. Hingegen wurde insbesondere die Mortalität der Arve (4\% Überlebende im Jahr 2010) sowohl auf spät ausapernden, kühlen Kleinstandorten (durch Gremeniella abietina) wie auch auf wärmeren, aber im Winter schneebedeckten Kleinstandorten (durch Phacidium infestans) stark durch Schneepilze bestimmt. Die Arve überlebte vor allem auf Kuppen und früh ausapernden Kleinstandorten, wo sie auch natürlicherweise verbreitet ist (Brücker 2010). 35 Jahre nach der Pflanzung waren die Bäume erst im unteren Teil der Aufforstung (unter $2150 \mathrm{~m}$ ü. M.) bereits so hoch, dass sie die zweifache durchschnittliche Schneehöhe überragten. Weiter oben ist die Schutzwirksamkeit gemäss diesem Kriterium noch nicht überall ausreichend. Die Lawinenverbauungen im nordwestlichen Teil der Stillberg-Aufforstung hatten bisher einen positiven Einfluss auf das Wachstum (weniger Stammbrüche durch Schneebewegungen), aber einen negativen auf das Überleben der Bäume (wegen späterer Ausaperung; Dellagiovanna 2011).

Der seit 2001 experimentell simulierte Klimawandel wirkte sich unterschiedlich auf die Baumarten aus. So zeigten bei erhöhtem $\mathrm{CO}_{2}$-Angebot die
Lärchen ein um rund 20\% stärkeres Wachstum, während die Bergföhren ihr Wachstum aufgrund von anderen limitierenden Faktoren nicht steigern konnten (Dawes et al 2011). Hingegen profitierten die Bergföhren vom erwärmten Boden, während die Lärchen nicht darauf reagierten. Einige experimentell behandelte Lärchen wiesen aber unter erhöhten $\mathrm{CO}_{2}$-Gehalten Frostschäden auf, weil sie im Frühjahr eine Woche früher austrieben (Martin et al 2010). Bei einer Erwärmung um $3{ }^{\circ} \mathrm{C}$ setzten die im Boden aktiveren Mikroorganismen zusätzliche Mengen $\mathrm{CO}_{2}$ frei, die nicht durch eine verstärkte $\mathrm{CO}_{2}$-Aufnahme aufgrund eines erhöhten Pflanzenwachstums ausgeglichen wurden. Eine substanzielle Menge Kohlenstoff gelangt somit aus dem Boden als $\mathrm{CO}_{2}$ in die Atmosphäre (Hagedorn et al 2010). Damit würden Waldgrenzenökosysteme wie der Stillberg bei der erwarteten Klimaänderung zumindest anfänglich nicht zu einer $\mathrm{CO}_{2}$-Senke, sondern zu einer $\mathrm{CO}_{2}$-Quelle.

Die Saatversuche am Stillberg und oberhalb davon zeigten, dass die sieben gesäten Baumarten mit Ausnahme der Grünerle (der es dort kleinstandortbedingt zu trocken war) selbst auf einer Höhe von $2400 \mathrm{~m}$ ü. M. gut keimten und überlebten. Dies war insbesondere bei der Waldföhre erstaunlich, welche in der Landschaft Davos normalerweise nur unterhalb von $1500 \mathrm{~m}$ ü. M. vorkommt. Mit steigender Meereshöhe nahm der Anteil der unterirdischen Biomasse zu, während die Gesamtbiomasse abnahm oder gleich blieb. Einen wichtigen Einfluss auf Wachstum und Biomassenverteilung der Sämlinge hatte die Stickstoffverfügbarkeit. Dies ist ein weite- 
rer Hinweis darauf, dass zusätzlich zur Temperatur noch weitere Faktoren wichtig für die Etablierung und das Wachstum von Bäumen an der oberen Baumgrenze sind.

\section{Wechselwirkungen zwischen Wald und Lawinen}

Ob eine bereits angerissene Lawine durch den Wald noch gebremst werden kann, hängt in erster Linie davon ab, wie gross die Beschleunigungsdis$\operatorname{tanz}$ in einer Waldlücke beziehungsweise oberhalb des Waldes ist. Grössere Lawinen, welche mehr als etwa $150 \mathrm{~m}$ oberhalb des Waldes anreissen, gewinnen bis zum Auftreffen auf den Wald so viel Energie, dass die Waldstruktur weiter unten kaum einen Einfluss auf die Auslaufdistanz hat. Bei kleineren Waldlawinen hat hingegen die Stammzahl im Bereich des Anrissgebietes einen signifikanten Einfluss auf die Auslaufdistanz. Interessant ist die Feststellung, dass vor allem die Stammzahl von kleinen Bäumen ( $<15 \mathrm{~cm}$ Brusthöhendurchmesser [BHD]) im Anrissgebiet die Auslaufdistanz und damit das Gefährdungspotenzial einer Lawine reduziert (Teich et al 2012a). Dies kann vor allem damit erklärt werden, dass kleine Bäume mit Ästen bis zum Boden die Terrainrauigkeit erhöhen und die Lawinenmasse verringern.

Wiederholungserhebungen in ehemaligen Anrissgebieten von Waldlawinen aus den 1980erJahren zeigen, dass einige der ehemaligen Anrissflächen heute dichter bestockt sind und dass insbesondere die Dichte von Bäumen geringer Dimension (4-15 cm BHD) zugenommen hat. Auch wenn Kronendeckungsgrad und Lückengrösse vielfach noch immer ein Anreissen von Lawinen unter extremen Bedingungen erlauben, kann angenommen werden, dass die Auslaufdistanz derselben im Vergleich zu früher kürzer ist. Allerdings hat sich der Wald in den ehemaligen Anrissflächen sehr unterschiedlich entwickelt. Wo es Baumstrünke und andere die Geländerauigkeit fördernde Elemente gab, haben sich die
Verjüngung und die Schutzwirksamkeit vielfach verbessert, in glatten Muldenlagen jedoch kaum.

Wetter- und Schneebedingungen während Waldlawinenabgängen konnten aufgrund von Clusteranalysen in zwei Kategorien eingeteilt werden (Teich et al 2012b): 1) Neuschnee-Waldlawinen, welche typischerweise während Perioden mit starkem Schneefall, wenig Sonneneinstrahlung, viel Wind und tiefen Temperaturen anreissen, und 2) andere Waldlawinen, die meist nach Perioden mit ergiebiger Sonneneinstrahlung, Erwärmung und nasser Schneedecke beobachtet wurden. Im Vergleich zu Freilandlawinen gehen Waldlawinen meist bei mächtigeren Schneedecken ab, da erst dann der Einfluss der Bodenrauigkeit im Wald verringert ist. In den letzten 41 Jahren (seit genügend zuverlässige Messdaten vorhanden sind) hat die Anzahl von potenziellen Waldlawinentagen, d.h. Tagen, die sich durch Waldlawinen begünstigende Schnee- und Witterungsbedingungen auszeichnen, abgenommen (Abbildung 4), und zwar sowohl für NeuschneeWaldlawinen (an 11 von 14 Messstationen, bedingt durch eine Abnahme von kalten Schneefallperioden) als auch für andere Waldlawinen (an 12 von 14 Messstationen, bedingt durch eine Abnahme der Schneedeckenmächtigkeit in tieferen Lagen).

\section{Waldentwicklung und Ökosystemleistungen}

Die Waldfläche in der Landschaft Davos hat zwischen 1954 und 2000 um 13\% zugenommen und der Anteil der geschlossenen Wälder (Schlussgrad locker bis gedrängt) ist von rund 33\% auf 54\% angestiegen (Tabelle 1; Lardelli 2003). Am stärksten waren die Waldzunahme und -verdichtung im Höhenbereich zwischen 1600 und $2000 \mathrm{~m}$ ü. M., also rund 100 bis $500 \mathrm{~m}$ unterhalb der aktuellen Waldgrenze (Kulakowski et al 2011; Abbildung 1). In die Zukunft projiziert ergibt sich daraus bis zum Jahr 2050 eine weitere Waldzunahme um 13\% im Trendszenario beziehungsweise um 21\% im Erwärmungsszenario. Beim Trendszenario ist dieser zusätzliche
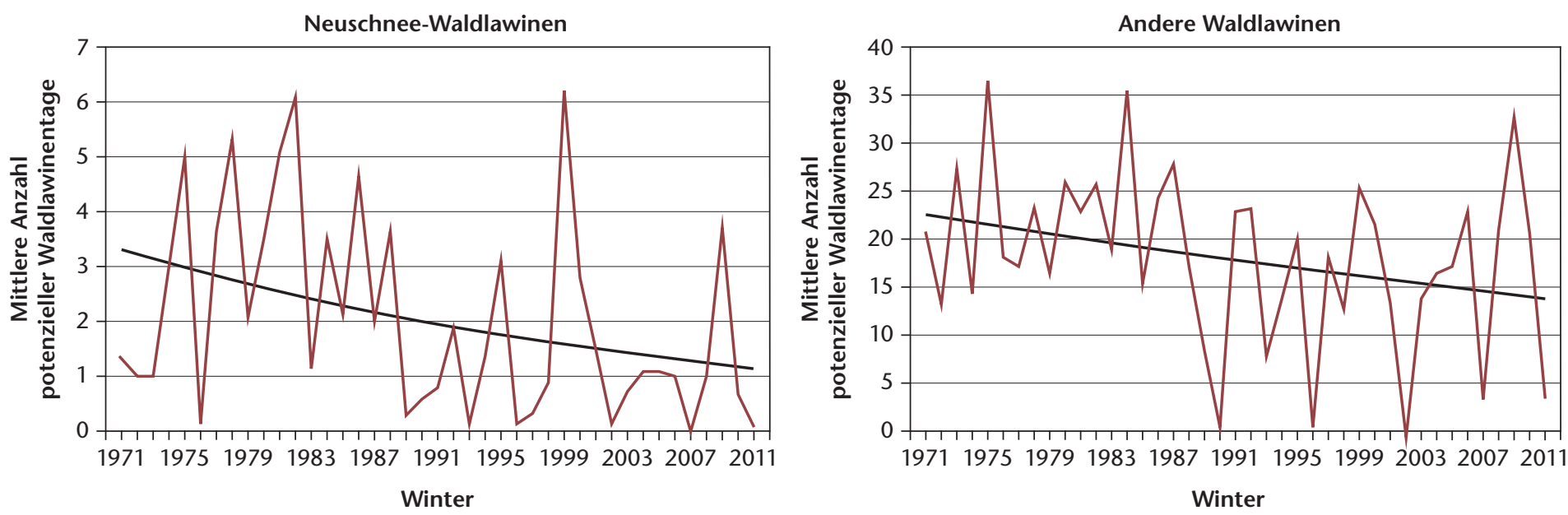

Abb 4 Entwicklung der Häufigkeit von potenziellen Waldlawinentagen gemittelt über 14 Schnee- und Wetterstationen im Schweizer Alpenraum, aufgeteilt in Neuschnee-Waldlawinen (links) und andere Waldlawinen (rechts), zwischen 1971 und 2011. 


\begin{tabular}{|l|c|c|c|c|}
\hline Schlussgrad & $\begin{array}{c}\text { Fläche 1954 } \\
\text { (ha) }\end{array}$ & $\begin{array}{c}\text { Fläche 2000 } \\
\text { (ha) }\end{array}$ & $\begin{array}{c}\text { Trendszenario } \\
\mathbf{2 0 5 0} \\
\text { (ha) }\end{array}$ & $\begin{array}{c}\text { Erwärmungs- } \\
\text { szenario 2050 } \\
\text { (ha) }\end{array}$ \\
\hline gedrängt bis locker & 1340 & 2416 & 3447 & 4085 \\
\hline räumig & 1502 & 1013 & 1316 & 1085 \\
\hline aufgelöst & 1155 & 1070 & 322 & 252 \\
\hline Total & 3997 & $\mathbf{4 4 9 9}$ & $\mathbf{5 0 8 5}$ & $\mathbf{5 4 2 2}$ \\
\hline
\end{tabular}

Tab 1 Schlussgrad der Waldbestände sowie Umfang der Waldfläche im Untersuchungsgebiet (ca. 90\% der Gemeinde Davos) in den Jahren 1954, 2000 und projiziert anhand von zwei Szenarien für das Jahr 2050. Die Schlussgrade «locker» bis «gedrängt» gemäss Schweizerischem Landesforstinventar (Brändli 2010) wurden zusammengefasst und als «geschlossener Wald» betrachtet.

\begin{tabular}{|l|c|c|c|}
\hline Ökosystemleistung & $\begin{array}{c}\text { Bewertung 2000 } \\
\text { (in Tausend CHF/Jahr) }\end{array}$ & $\begin{array}{c}\text { Bewertung Trend- } \\
\text { szenario 2050 } \\
\text { (in Tausend CHF/Jahr) }\end{array}$ & $\begin{array}{c}\text { Bewertung } \\
\text { Erwärmungssenario } \\
\text { 2050 }\end{array}$ \\
(in Tausend CHF/Jahr)
\end{tabular}

Tab 2 Bewertung ausgewählter Ökosystemleistungen in der Landschaft Davos für das Jahr 2000 und anhand zweier verschiedener Szenarien für das Jahr 2050.

Wald vor allem auf weitere landwirtschaftliche Extensivierungen zurückzuführen, beim Erwärmungsszenario ergeben sich insbesondere in den höheren Lagen zusätzliche Waldverdichtungen und Waldausdehnungen.

Der Gesamtwert der fünf für die Landschaft Davos modellierten Ökosystemleistungen (Lawinenschutz, Erholung, C-Speicherung, Auerhuhnhabitate und Holzproduktion) steigt von rund $106 \mathrm{Mio}$. CHF pro Jahr im Jahr 2000 auf rund 130 Mio. CHF pro Jahr (+23\%) beim Trendszenario respektive auf rund 135 Mio. CHF pro Jahr (+27\%) beim Erwärmungsszenario (Tabelle 2). Dieser Anstieg ist einerseits auf die Ausdehnung der Waldfläche zurückzuführen (Ökosystemleistungen ausserhalb des Waldes werden hier nicht berücksichtigt). Andererseits tragen auch Waldstrukturveränderungen und erwartete Werterhöhungen einzelner Ökosystemleistungen (z.B. die Erhöhung von $\mathrm{CO}_{2}$-Emissionsabgaben, des Holzpreises oder des prognostizierten Erholungswerts) zur Erhöhung des Gesamtwertes bei. Diese beiden Faktoren führen zusammen beispielsweise zu einer Erhöhung des Werts der Erholungsleistung um 50\%.

Den mit Abstand grössten Wert heute und in Zukunft zeigt die Ökosystemleistung «Lawinenschutz». Die Ökosystemleistung «Holzproduktion» beeinflusst hingegen den Gesamtwert in allen Fällen negativ (Tabelle 2). Dabei reagiert der finanzielle Erfolg dieser Ökosystemleistung sensitiv auf Annahmen bezüglich des Holzpreises. Bei einem heute realistischen durchschnittlichen Holzpreis von CHF 96.- $/ \mathrm{m}^{3}$ und bei einem für 2050 prognostizierten Holzpreis von CHF 115.-/m³ ist die Holzproduk- tion (isoliert betrachtet, ohne Querfinanzierung durch die Bereitstellung von Lawinenschutz und anderen Waldleistungen) in den meisten Davoser Waldbeständen unrentabel, was sich in negativen Werten für die Ökosystemleistung «Holzproduktion» ausdrückt (Tabelle 2). Wenn sich der Holzpreis hingegen auf CHF 120.-/ $\mathrm{m}^{3}$ erhöhen würde, wäre sie in $80 \%$ der Davoser Wälder auch ohne Querfinanzierung gewinnbringend.

Der Wert der einzelnen Ökosystemleistungen unterscheidet sich je nach Topografie, Standort und Waldstruktur stark. Auf rund 50\% der Davoser Waldfläche ist der Lawinenschutz die wertmässig wichtigste Waldleistung (Abbildung 5). Im Durchschnitt beläuft sich ihr Wert auf rund CHF 30000.-/ha. Je nach Topografie und Schadenpotenzial im Einzugsgebiet variiert der berechnete Wert aber zwischen CHF 3.-/ha und CHF 698000.-/ha. Die potenziellen Auerhuhnhabitate konzentrieren sich wegen spezifischer Ansprüche bezüglich Waldstruktur und Topografie auf eine relativ kleine Fläche von rund 1400 ha, sind aber als Folge der modellierten Waldflächenzunahme eher grösser als heute (Tabelle 2). Im Vergleich zu den Leistungen «Lawinenschutz» und «Auerhuhnhabitate» sind die Erholungsleistung und die C-Speicherung gleichmässiger auf alle Wälder verteilt, obwohl auch dort räumliche Unterschiede in der Höhe der Leistungen bestehen (Abbildung 5).

Mehr als 65\% aller Davoser Wälder weisen Überlappungen von drei oder mehr der betrachteten Ökosystemleistungen auf (Abbildung 5). Wichtige Synergien bestehen darin, dass durch die Pflege des Schutzwaldes gleichzeitig auch Holz produziert werden kann. Je nach Waldstruktur bestehen auch Synergien zwischen Lawinenschutz und Auerhuhnhabitaten oder C-Speicherung. Potenzielle Konflikte existieren am ehesten im Zusammenhang mit der Erholungsleistung, da von Erholungsuchenden eher offene Wälder bevorzugt werden und andere Ökosystemleistungen wie Lawinenschutz oder C-Speicherung (zumindest über einen gewissen Zeitraum betrachtet) an Wert gewinnen, wenn der Wald dichter wird. In den Zukunftsszenarien ändern sich die Synergien und Zielkonflikte zwischen den verschiedenen Waldleistungen nicht wesentlich.

\section{Diskussion}

\section{Veränderung der Waldfläche und des Wald- aufbaus}

Sowohl die am Stillberg durchgeführten Experimente als auch die verwendeten Modelle deuten darauf hin, dass in der hochalpinen Region Davos die Waldfläche auch in Zukunft weiter zunehmen wird. Allerdings zeigen die Experimente ebenso, dass an einer grundsätzlich durch die Kälte limitierten Waldgrenze nebst Temperaturerhöhungen auch noch 


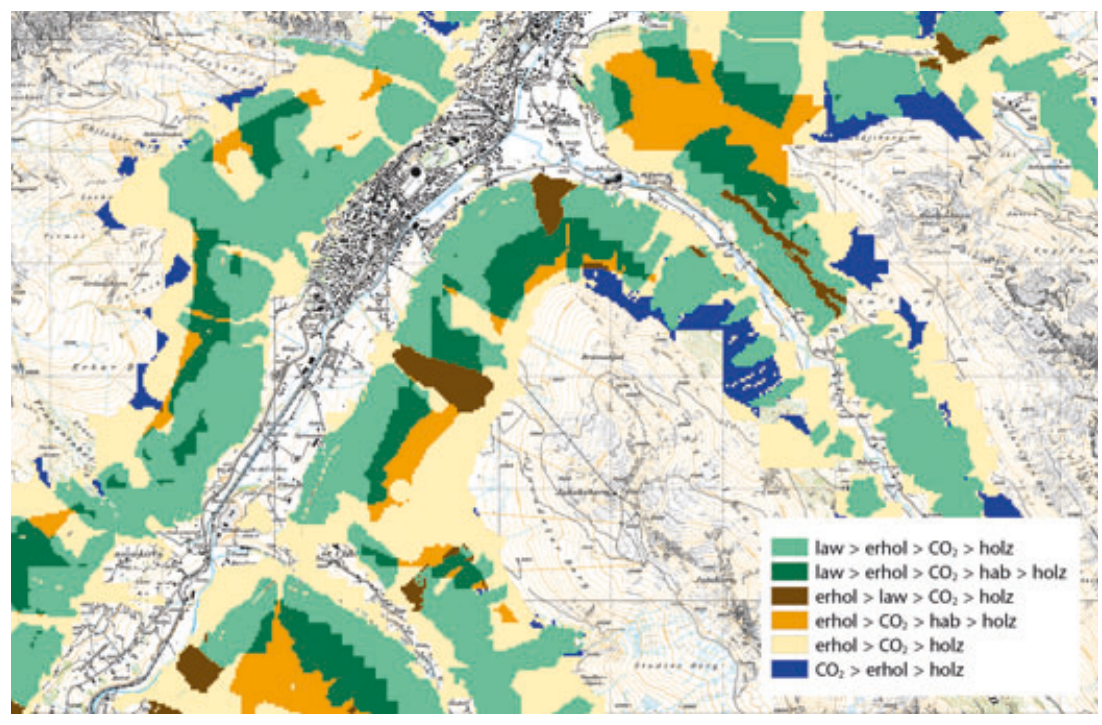

Abb 5 Räumliche Verteilung der Ökosystemleistungen nach ihrem Wert in einem Ausschnitt des Davoser Waldes im Erwärmungsszenario für das Jahr 2050. law: Lawinenschutz, erhol: Erholung, $\mathrm{CO}_{2}$ : C-Speicherung, hab: Auerhuhnhabitate und holz: Holzproduktion.

viele andere Faktoren das Baumwachstum und die Mortalität beeinflussen und dass in verschiedenen Stadien der Baumverjüngung unterschiedliche Faktoren wichtig sein können (Barbeito et al 2012, Moos 2012). Dazu tragen auch Faktoren bei, welche bei den Versuchen am Stillberg nicht berücksichtigt wurden, wie beispielsweise dichte Zwergstrauchheiden oder Beweidung (Ponge et al 1998, Motta et al 2006).

Aufgrund der Resultate der Aufforstung am Stillberg hat die Pionierbaumart Lärche das grösste Potenzial, sich oberhalb der jetzigen Waldgrenze zu etablieren und von den höheren $\mathrm{CO}_{2}$-Konzentrationen zu profitieren. Allerdings zeigt die Wiederholung einer Kartierung der obersten Waldgrenzenbäume im Dischmatal (Walder 1983), dass sich während der letzten 40 Jahre nebst der Lärche vor allem auch die Arve auf für sie günstigen Kleinstandorten und - weniger ausgeprägt - die als weniger frosttolerant bekannte Fichte etablieren konnten (Leibold 2012). Diese Untersuchungen bestätigen Modellresultate, welche besagen, dass ein wärmeres Klima langfristig zu einem Anstieg der kältelimitierten Waldgrenzen führen muss und dass auch Artverschiebungen an der oberen Waldgrenze nicht ausgeschlossen sind (Schuhmacher \& Bugmann 2006). Sie zeigen aber auch, dass der Anstieg der Waldgrenze zögerlich und räumlich differenziert erfolgt, und dies insbesondere dort, wo auch in den nächsten Jahrzehnten noch schneereiche Winter zu erwarten sind und wo die komplexe Topografie und Nutzungsgeschichte unterschiedlich günstige Verjüngungsbedingungen schafft.

Die Untersuchungen lassen erwarten, dass die stärksten Änderungen nicht oberhalb der jetzigen Waldgrenze, sondern innerhalb des heutigen Waldareals ablaufen werden. Dank den wärmeren Bedingungen und der Aufgabe der Beweidung werden die ehemals offenen Fichten- und Lärchenwälder zuneh- mend dichter. Viele dieser Wälder sind bereits heute in einer frühen Phase der Selbstdifferenzierung, in der zunehmend Konkurrenz herrscht und sich Totholz anreichert (Krumm et al 2012). Die weitere Entwicklung kann mithilfe der hier verwendeten Modelle nur schwer abgeschätzt werden, da sie sowohl von waldbaulichen Eingriffen als auch von natürlichen Störungen wie Schneebruch, Windwurf, Feuer und Borkenkäferkalamitäten abhängt. Bei zunehmender Erwärmung und Sommertrockenheit sowie fortschreitender Zunahme des Schlussgrades der Waldbestände muss mit einer Zunahme solcher Störungen gerechnet werden (Wohlgemuth et al 2008). Dies gilt insbesondere für Borkenkäferbefall, welcher bisher in Davos wegen zu tiefer Sommertemperaturen nicht grossflächig auftrat, bei einer Temperaturzunahme von 2 bis $4{ }^{\circ} \mathrm{C}$ aber deutlich häufiger werden könnte (Wermelinger \& Seifert 1998). Auch Waldbrände, welche bisher nur ausnahmsweise nach warmen und trockenen Witterungsperioden im Sommer auftraten, könnten in Zukunft häufiger werden. Wie stark und wo die Davoser Wälder durch solche Störungen betroffen sein werden, ist offen. Zwar wirkt die komplexe, mit verschiedenen Ausbreitungsbarrieren durchsetzte Topografie grossflächigen Störungen entgegen. Trotzdem sind es vor allem die eher grossflächig wirkenden Störungen wie Borkenkäferbefall, Waldbrand und Windwurf, welche der insgesamt positiven Wertentwicklung der Waldleistungen in der Landschaft Davos in den nächsten Jahrzehnten am stärksten entgegenwirken könnten (vgl. auch Schuhmacher \& Bugmann 2006).

\section{Veränderung der Ökosystemleistungen}

Gemäss der hier durchgeführten Monetarisierung ist der Lawinenschutz mit Abstand die wertvollste Waldleistung im Raum Davos, und 50\% des Waldes dienen vorrangig dem Lawinenschutz. Auch wenn die für Waldlawinen kritischen Schnee- und Witterungsbedingungen in Zukunft tendenziell weniger häufig auftreten dürften und sich die Schutzwirkung in den letzten Jahrzehnten eher verbessert hat, muss der Erfüllung der Lawinenschutzleistung (und der Schutzleistung gegenüber anderen Naturgefahren, welche hier nicht bewertet wurde) auch in Zukunft grösste Beachtung geschenkt werden. Unsere Auswertungen deuten darauf hin, dass der Förderung von Verjüngung und Bodenrauigkeit (Strünke und gesicherte Baumstämme) in Lawinenanrissgebieten noch mehr Gewicht beigemessen werden sollte.

Zukunftsszenarien mit einwachsendem Schutzwald auf bisher landwirtschaftlich genutzten Flächen weisen zudem auf Potenziale hin, die auf einer besseren Abstimmung der landwirtschaftlichen und forstlichen Förderungsmassnahmen beruhen und die zu einer Verbesserung der Ökosystemleistungen insgesamt führen. Während die Ökosystemleistung «Lawinenschutz» am besten mit einer möglichst 
dauerhaften Bestockung ohne grosse Lücken erbracht wird, sind für andere Leistungen variablere Bestockungsziele möglich. Im Sinn einer Gesamtoptimierung der Ökosystemleistungen könnten daher auch ein stärkerer Vorratsabbau und eine aktive Verhinderung von Vorratszunahmen in einem Teil der Davoser Wälder ins Auge gefasst werden. Dies wäre insbesondere ein Beitrag zur Erhöhung der Erholungsleistung sowie zur Verbesserung der Ökosystemleistung «Holzproduktion», welche zurzeit fast nur im Zusammenhang mit anderen Waldökosystemleistungen rentabel ist. Daneben kann ein Vorratsabbau respektive die Verhinderung einer Vorratszunahme auch als eine Massnahme zur Anpassung an den Klimawandel betrachtet werden, da so grossflächigen Störungen entgegengewirkt werden kann.

Möglichkeiten und Grenzen der Modellierung In das hier verwendete Modellsystem lassen sich auch weitere Ökosystemleistungen integrieren und damit bewerten. Darauf aufbauend könnte eine in der Praxis anwendbare Entscheidungshilfe für die multifunktionale Bewirtschaftung von Gebirgsregionen entwickelt werden. Für eine umfassendere Betrachtung wäre es zunächst sicher sinnvoll, auch die Leistungen von Graslandökosystemen (also des Nichtwaldes) zu berücksichtigen. Zudem besteht bei einigen der fünf verwendeten Bewertungsansätze noch Verbesserungspotenzial. So berücksichtigen beispielsweise die Modelle zur C-Speicherung noch nicht die neuen Erkenntnisse der Stillberg-Experimente, wonach die Bodenatmung bei höheren Bodentemperaturen die Biomassenzunahme im Waldgrenzenbereich zumindest teilweise kompensieren kann (Hagedorn et al 2010). Auch deckt das Auerhuhn - wenn auch eine gute Indikatorart für strukturreiche Wälder - nicht sämtliche Habitatbedürfnisse der verschiedenen Pflanzen- und Tierarten im Wald ab, und die Erholungsleistung wurde mit einem einfachen Modell bewertet, welches vor allem auf der Annahme beruht, dass offene und gut zugängliche Wälder für die Erholungsnutzung wertvoller sind.

Trotz solchen Einschränkungen stellt das angewendete Bayes'sche Netzwerk dank der Integration von Expertenwissen und der Berücksichtigung von Unsicherheiten bereits eine methodische Weiterentwicklung von bisherigen Modellsystemen dar (Grêt-Regamey et al 2012). Um aber ein Modellsystem, welches über die zukünftige Entwicklung von Ökosystemen und deren Leistungen Auskunft geben soll, weiter zu verbessern, ist es vor allem nötig, dass - dem Ansatz des «Mountland»-Projektes folgend - die einzelnen Modelle auf verschiedenen räumlichen und zeitlichen Skalen immer wieder mit Felddaten und Experimenten kombiniert und verifiziert werden.

Eingereicht: 4. April 2012, akzeptiert (mit Review): 5. Oktober 2012

\section{Dank}

Wir danken allen Forschenden und Technikern, die an den Untersuchungen in der Landschaft Davos und insbesondere am Stillberg beteiligt waren, sowie den Experten, welche zur Verbesserung der Modellierung der Ökosystemleistungen beigetragen haben. Für wertvolle Hinweise zum Manuskript danken wir Peter Brang, Georg Leitinger, Andreas Rigling sowie den zuständigen Förstern Hanspeter Hefti und Andreas Kessler.

\section{Literatur}

BARBEITO I, DAWES M, RIXEN C, SENN J, BEBI P (2012) Factors driving survival and growth at treeline: a 30 -year experiment of 92,000 trees. Ecology 93: 389-401.

BEBI P, KULAKOWSKI D, RIXEN C (2009) Snow avalanche disturbances in forest ecosystems - state of research and implications for management. For Ecol Manage 257: 1883-1892.

BECK C (2008) Literaturstudie zur monetären Bewertung von Nicht-Holzleistungen des Waldes. In: Moser TA et al, editors. Inwertsetzung von Waldwerten und Waldleistungen. Ergänzungsbeitrag zur Cost Aktion E45. Zürich: Eidg Techn Hochschule, Professur Umweltpolitik Umweltökonomie. pp. 89-196.

BERNASCONI A, SCHROFF U, AYÙS PELLITERO T (2005) Erholung und Walddynamik. Synthesebericht. http://afw-ctf.ch/walddynamik_synthese.pdf (1.10.2012).

BRÄNDLI UB, EDITOR (2010) Schweizerisches Landesforstinventar. Ergebnisse der dritten Erhebung 2004-2006. Birmensdorf: Eidgenöss Forsch.anstalt WSL. 312 p.

BRÜCKER R (2010) Tree mortality caused by snow fungi near treeline. Zürich: Univ Zürich, Masterarbeit. $59 \mathrm{p}$.

CHRISTEN M, KOWALSKI J, BARTELT P (2011) RAMMS: Numerical simulation of dense snow avalanches in three-dimensional terrain. Cold Reg Sci Technol 65: 273-273.

DAWES MA ET AL (2011) Species-specific tree growth responses to 9 years of $\mathrm{CO}_{2}$ enrichment at the alpine treeline. J Ecol 99: 383-394.

DELLAGIOVANNA M (2011) Lawinenschutzwirksamkeit der Hochlagenaufforstung Stillberg. Zürich: ETH Zürich, Masterarbeit. $66 \mathrm{p}$.

GRAF RF, BOLLMANN K, SUTER W, BUGMANN H (2005) The importance of spatial scale: Capercaillie in the Swiss Alps. Landsc Ecol 20: 703-717.

GRÊT-REGAMEY A, BEBI P, BISHOP ID, SCHMID WA (2008) Linking GIS-based models to value ecosystem services in an $\mathrm{Al}$ pine region. J Environ Manage 89: 197-208.

GRÊT-REGAMEY A, STRAUB D (2006) Spatially explicit avalanche risk assessment linking Bayesian networks to a GIS. Nat Hazards Earth Syst Sci 6: 911-926.

GRÊT-REGAMEY A, BRUNNER SH, ALTWEGG JM, BEBI P (2012) Facing uncertainty in ecosystem services-based resource management. J Environ Manage. doi: 10.1016/j.jenvman.2012.07.028

HAGEDORN F ET AL (2010) Short-term responses of ecosystem carbon fluxes to experimental soil warming at the Swiss alpine treeline. Biogeochemistry 97: 7-19.

HUBER UM, BUGMANN H, REASONER MA (2005) Global change and mountain regions. An overview of current knowledge. Dordrecht: Springer. $650 \mathrm{p}$.

KRUMM F, KULAKOWSKI D, RISCH A, BRÄNDLI UB, BEBI P (2012) Stem exclusion and mortality in unmanaged subalpine forests of the Swiss Alps. Eur J For Res 131: 1571-1583.

KULAKOWSKI D, BEBI P, RIXEN C (2011) The interacting effects of land use change, climate change and suppression of natural disturbances on landscape forest structure in the Swiss Alps. Oikos 120: 216-225. 
LARDELLI C (2003) Dynamik und Stabilität von Lawinenschutzwäldern: eine Luftbild- und GIS-gestützte Analyse. Zürich: Univ Zürich, Diplomarbeit. 105 p.

LEILBOLD E (2012) Wiederaufnahme von Jungbäumen oberhalb der aktuellen Waldgrenze im Dischmatal nach 40 Jahren. Wien: Univ Bodenkultur, Masterarbeit. $54 \mathrm{p}$.

MARTIN MA, GAVAZOV K, HÄTTENSCHWILER, S, KÖRNER C RIXEN C (2010) Reduced early growing season freezing resistance in alpine treeline plants under elevated atmospheric $\mathrm{CO}_{2}$. Glob Chang Biol 16: 1057-1070.

MEYER-GRASS M, SCHNEEBELI M (1992) Die Abhängigkeit der Waldlawinen von Standorts-, Bestandes- und Schneeverhältnissen. In: Fiebiger G, Zollinger F, editors. Schutz des Lebensraumes vor Hochwasser, Muren und Lawinen. Klagenfurt: Forschungsgesellschaft vorbeugende Hochwasserbekämpfung, Tagungspublikation Internationales Symposium Interpraevent 1992, Band 2. pp. 443-455.

MOOS C (2012) Wachstum von Sämlingen sieben verschiedener Baumarten an und über der Baumgrenze in den Schweizer Alpen. Zürich: ETH Zürich, Bachelorarbeit. $80 \mathrm{p}$.

MOTTA R, MORALES M, NOLA P (2006) Human land-use, forest dynamics and tree growth at the treeline in the Western Italian Alps. Ann For Sci 63: 739-747.

NELSON E ET AL (2009) Modeling multiple ecosystem services, biodiversity conservation, commodity production and tradeoffs at landscape scales. Front Ecol Environ 7: 4-11.

OCCC (2003) Extreme events and climate change. Bern: OcCC. $88 \mathrm{p}$.

PONGE JF ET AL (1998) The forest regeneration puzzle. Biological mechanisms in humus layer and forest vegetation dynamics. BioScience 48: 523-530.

SCHÖNENBERGER W, FREY W (1988) Untersuchungen zur Ökologie und Technik der Hochlagenaufforstung. Forschungser-

\section{Veränderung von Wald und Waldleistungen in der Landschaft Davos im Zuge des Klima- wandels}

Die Auswirkungen des Klimawandels auf die Wälder von Davos wurden mit Felduntersuchungen an der Waldgrenze, Analysen zur Lawinenschutzfunktion und räumlich expliziten Modellen zur Bereitstellung von Ökosystemleistungen untersucht. Aufgrund von Langzeitbeobachtungen und Experimenten auf der Versuchsfläche Stillberg zeigte sich, dass Waldgrenzenbäume artspezifisch auf eine $\mathrm{CO}_{2}$-reichere Atmosphäre und eine wärmere Zukunft reagieren. Wachstum und Mortalität der im Jahr 1975 an der Waldgrenze gepflanzten Bäume waren dabei stark durch die Schneebedeckung und andere kleinräumig variierende Faktoren bestimmt. Dies weist zusammen mit anderen Felduntersuchungen in der Region darauf hin, dass die Waldgrenze zögerlich und zunächst nur auf begünstigten Kleinstandorten ansteigen wird. Aufgrund der Modellierungen wird damit gerechnet, dass auch im Jahr 2050 der Lawinenschutz die wichtigste Waldfunktion in Davos sein wird, auch wenn die für das Anreissen von Waldlawinen kritischen Witterungsbedingungen in den letzten 40 Jahren eher seltener geworden sind. Es wird zudem erwartet, dass einige der heute noch lückigen Wälder sich schliessen dürften, was bezüglich Lawinenschutz positiv zu werten ist. Einbussen in der Schutzwirkung könnten sich in Zukunft aber durch die Zunahme von natürlichen Störungen wie Waldbränden und Borkenkäferkalamitäten ergeben. Die Quantifizierung und räumliche Überlagerung von fünf Waldleistungen, die für die Landschaft Davos von Bedeutung sind (Lawinenschutz, Erholung, C-Speicherung, Auerhuhnhabitate, Holzproduktion), deuten eher auf eine Zunahme des Werts von Waldleistungen hin. gebnisse aus dem Lawinenanrissgebiet Stillberg. Schweiz Z Forstwes 139: 735-820.

SCHUMACHER S, BUGMANN H (2006) The relative importance of climatic effects, wildfires and management for future forest landscape dynamics in the Swiss Alps. Glob Chang Biol 12: 1435-1450.

TEICH M, BARTELT P, GRÊT-REGAMEY A, BEBI P (2012A) Snow avalanches in forested terrain: Influence of forest parameters, topography and avalanche characteristics on runout distance. Arct Antarc Alp Res. doi: 10.1657/1938-4246-44.4

TEICH M, MARTY C, GOLLUT C, GRÊT-REGAMEY A, BEBI P (2012B) Snow and weather conditions associated with avalanche releases in forests: Rare situations with decreasing trends during the last 41 years. Cold Reg Sci Technol 83: 77-88.

THÜRIG E, SCHMID S (2008) Jährliche $\mathrm{CO}_{2}$-Flüsse im Wald: Berechnungsmethode für das Treibhausgasinventar. Schweiz Z Forstwes 159: 31-38. doi: 10.3188/szf.2008.0031

ULRICH M (2008) Strukturerfassung und -dynamik von waldgrenzennahen Wäldern mit verminderter Lawinenschutzwirksamkeit in der Landschaft Davos. Wädenswil: Zürcher Hochschule angewandte Wissenschaften. 65 p.

WALDER U (1983) Ausaperung und Vegetationsverteilung im Dischmatal. Mitt Eidgenöss Forsch.anstalt Wald Schnee Landsch 59: 81-206

WERMELINGER B, SEIFERT M (1998) Analysis of the temperature dependent development of the spruce bark beetle Ips typographus (L.) (Col., Scolytidae). J Appl Entomol 122: 185-191.

WOHLGEMUTH T ET AL (2008) Effekte des Klimawandels auf Windwurf, Waldbrand und Walddynamik im Schweizer Wald. Schweiz Z Forstwes 159: 326-334. doi: 10.3188/szf.2008.0326

ZAPPA M, POS F, STRASSER U, WARMERDAM P, GURTZ J (2003) Seasonal water balance of an Alpine catchment as evaluated by different methods for spatially distributed snowmelt modelling. Nord Hydrol 34: 179-202.

\section{Modification de la forêt et de ses prestations dans le paysage davosien sous l'effet du changement climatique}

Les effets du changement climatique sur les forêts de Davos ont été l'objet d'examens sur le terrain à la limite forestière, d'analyses de la fonction protectrice contre les avalanches et de modélisations spatialement explicites des prestations écosystémiques. Des observations à long terme et des expérimentations sur l'aire d'essais de Stillberg révèlent que les arbres situés à la limite forestière réagissent spécifiquement à une atmosphère plus riche en $\mathrm{CO}_{2}$ et à un environnement plus chaud. Par ailleurs, le manteau neigeux et d'autres facteurs variant à petite échelle ont fortement influencé la croissance et la mortalité des arbres plantés à cette limite en 1975. II ressort de toutes ces investigations que la limite forestière ne s'élèvera que lentement et d'abord uniquement dans les microstations bénéficiant de conditions favorables. Les modélisations laissent présumer qu'en 2050 encore, la protection contre les avalanches sera la principale fonction de la forêt de Davos, même si les tendances du passé indiquent que les conditions atmosphériques critiques pour le déclenchement d'avalanches vont plutôt se raréfier. Il est en outre probable que quelques forêts, aujourd'hui clairiérées, tendront à se fermer, ce qui favorisera la protection contre les avalanches. Mais l'effet protecteur pourrait être atténué à l'avenir par l'augmentation des perturbations naturelles, comme les incendies de forêt et les fortes pullulations de bostryches. D'après une quantification et une superposition spatiale de cinq prestations forestières importantes pour le paysage davosien (protection contre les avalanches, détente, stockage du $\mathrm{CO}_{2}$, habitats du grand tétras et production ligneuse), la valeur de ces prestations devrait augmenter à l'avenir. 